\title{
RECONSTRUCTION IN TIME-WARPED WEIGHTED SHIFT-INVARIANT SPACES WITH APPLICATION TO SPLINE SUBSPACES
}

\author{
JUN XIAN and YONGJIN LI
}

Received 17 December 2002

\begin{abstract}
We discuss the reproducing kernel structure in shift-invariant spaces and the weighted shift-invariant spaces, and obtain the reconstruction formula in timewarped weighted shift-invariant spaces, then apply them to a spline subspace. In the spline subspace, we give a reconstruction formula in a time-warped spline subspace.
\end{abstract}

2000 Mathematics Subject Classification: 41A15, 42C15, 94A12.

1. Introduction. The problem of reconstruction of a function $f$ has been applied widely to signal or image processing, so it is of vital importance to study the subject in the field of signal or image processing. The problem of reconstruction means that we reconstruct the function $f$ on $\mathbb{R}^{d}$ from its countable samples. It is well known that in the sampling and reconstruction problem, the function $f$ is often assumed to belong to a shift-invariant space [1, 2, 5]. For instance,

$$
V^{2}(\sin c)=\left\{f=\sum_{k \in \mathbb{Z}} c(k) \sin c(\cdot-k):(c(k)) \in l^{2}\right\},
$$

which is the classical space of band-limited spaces, often used as a model in sampling theory. For the special shift-invariant subspaces, spline subspaces, there are many practical applications to signal or image processing. So the research of spline subspaces received much attention in $[1,6,7]$ and so on.

In [4], Clark et al. first introduced the concept of time-warped space and obtained the reconstruction formula in time-warped band-limited signals. They generalized the classical Shannon sampling theorem and provided a method of reconstruction for a certain space of non-band-limited signal from irregular spaced samples. Since we often deal with space of non-band-limited signal and irregular spaced samples in practical applications, Clark's method is very valuable.

In this paper, we discuss the reproducing kernel structure in shift-invariant spaces $V^{2}(\varphi)$ and time-warped shift-invariant spaces $V_{\gamma}^{2}(\varphi)$. Then we obtain the reconstruction formula in the time-warped weighted shift-invariant spaces 
$V_{m, \gamma}^{p}(\varphi)$. As a special example or a particular application, we obtain reconstruction formula in the time-warped spline subspaces. Then we use another method to obtain the reconstruction formula in time-warped spline subspaces. Moreover, we construct reproducing kernel in spline and time-warped spline subspaces. Because time-warped spaces are more general than the original spaces themselves, we generalize the results of $[2,3,5,7]$ with time-warped method.

2. Notations and definitions. Given a space $S$ of function $f: \mathbb{R}^{n} \rightarrow \mathbb{C}^{n}$ and an invertible continuous function $\gamma: \mathbb{R}^{n} \rightarrow \mathbb{R}^{n}$, the time-warped function space is $S_{\gamma}=\left\{f_{\gamma}: f_{\gamma}(t)=f \circ \gamma(t)=f(\gamma(t)), \forall t \in \mathbb{R}^{n}\right\}$, and $\gamma$ is called the warping function.

A reproducing kernel on a Hilbert space $H$ is a function $k: \mathbb{R}^{n} \times \mathbb{R}^{n} \rightarrow \mathbb{C}^{n}$ such that $k(\cdot, x) \in H$ and $f(x)=\langle f, k(\cdot, x)\rangle$ for every $x \in \mathbb{R}^{n}$ and $f \in H$. A weight is a nonnegative continuous function on $\mathbb{R}^{n}$. A weight $m$ is called $s$ moderate if there are constants $C, s \geq 0$ such that $m(t+x) \leq C(1+|t|)^{s} m(x)$ for all $t, x \in \mathbb{R}^{n}$. A function $f$ belongs to $L_{m}^{p}\left(\mathbb{R}^{n}\right)$ with weight function $m$ if $m f$ belongs to $L^{p}\left(\mathbb{R}^{n}\right)$. Equipped with the norm $\|f\|_{L_{m}^{p}}:=\|m f\|_{L^{p}}$, the space $L_{v}^{p}$ is a Banach space.

We also consider the weighted sequence spaces $l_{m}^{p}\left(\mathbb{Z}^{n}\right)$ with weight $m$. A sequence $\left\{\left(c_{k}\right): k \in \mathbb{Z}^{n}\right\}$ belongs to $l_{m}^{p}$ if $\left((\mathrm{cm})_{k}\right)=\left(c_{k} m_{k}\right)$ belongs to $l^{p}$ with norm $\|c\|_{l_{m}^{p}}:=\|m c\|_{l^{p}}$, where $\left(m_{k}\right)$ is the restriction of $m$ to $\mathbb{Z}^{n}$.

Shift-invariant spaces are defined by

$$
V^{p}(\varphi):=\left\{f=\sum_{k \in \mathbb{Z}^{d}} c(k) \varphi(\cdot-k): c \in l^{p}\right\} \subset L^{p}\left(\mathbb{R}^{d}\right) .
$$

Weighted shift-invariant spaces are defined by

$$
V_{m}^{p}(\varphi):=\left\{f=\sum_{k \in \mathbb{Z}^{d}} c(k) \varphi(\cdot-k): c \in l_{m}^{p}\right\} \subset L_{m}^{p}\left(\mathbb{R}^{d}\right) .
$$

Time-warped shift-invariant spaces are defined by

$$
V_{\gamma}^{p}(\varphi):=\left\{f_{\gamma}: f_{\gamma}(\cdot)=f(\gamma(\cdot)), f \in V^{p}(\varphi)\right\} .
$$

Time-warped weighted shift-invariant spaces are defined by

$$
V_{m, \gamma}^{p}(\varphi):=\left\{f_{\gamma}: f_{\gamma}(\cdot)=f(\gamma(\cdot)), f \in V_{m}^{p}(\varphi)\right\} .
$$

Spline subspace $V_{N}:=\left\{f=\sum_{k \in \mathbb{Z}} c_{k} \varphi_{N}(\cdot-k):\left\{c_{k}\right\} \in l^{2}\right\}$, where $\varphi_{N}=$ $\chi_{[0,1]} * \cdots * \chi_{[0,1]}(N$ convolution, $N \geq 1)$.

Two sets $\left\{\varphi_{n}\right\}$ and $\left\{\tilde{\varphi}_{n}\right\}$ are biorthogonal if $\left\langle\varphi_{n}, \tilde{\varphi}_{m}\right\rangle=\delta_{m n}$. If both $\left\{\varphi_{n}\right\}$ and $\left\{\tilde{\varphi}_{n}\right\}$ are the frames of some space $V$, and for any $f \in V$, the following 
equalities hold: $f=\sum_{k}\left\langle f, \varphi_{k}\right\rangle \tilde{\varphi}_{k}=\sum_{k}\left\langle f, \tilde{\varphi}_{k}\right\rangle \varphi_{k}$, then $\left\{\varphi_{n}\right\}$ and $\left\{\tilde{\varphi}_{n}\right\}$ are mutually called dual frames.

3. Reproducing kernel and basic properties in shift-invariant space and time-warped shift-invariant space. If $\varphi$ satisfies the following conditions:

(i) $\left\{\varphi(\cdot-k), k \in \mathbb{Z}^{d}\right\}$ form a Riesz basis for the Hilbert space $V^{2}(\varphi)$,

(ii) $\varphi$ is continuous,

(iii) $\varphi$ satisfies the decay condition $|\varphi(x)| \leq C(1+|x|)^{-d-s-\epsilon}$ for some $\epsilon>0$ and $s \geq 0$ such that the weight $m$ is $s$-moderate,

then for $p=2$ and $m=1$, there exists reproducing kernel $k(\cdot, x)$ such that $f(x)=\langle f, k(\cdot, x)\rangle$ for any $f \in V^{2}(\varphi)$ and $x \in \mathbb{R}^{n}[2,5]$.

Let $\left\langle f_{\gamma}, g_{\gamma}\right\rangle_{\gamma}=\langle f, g\rangle$ for any $f_{\gamma}, g_{\gamma} \in V_{\gamma}^{2}(\varphi)$, then $\langle\cdot, \cdot\rangle_{\gamma}$ is an inner product in $V_{\gamma}^{2}(\varphi)$, and the time-warped space $V_{\gamma}^{2}(\varphi)$ is a Hilbert space with reproducing kernel defined by $k_{\gamma}(w, x)=k(\gamma(w), \gamma(x))$.

In fact, for any $f_{\gamma} \in V_{\gamma}^{2}(\varphi), x \in \mathbb{R}^{n},\left\langle f_{\gamma}, k_{\gamma}(\cdot, x)\right\rangle_{\gamma}=\langle f, k(\cdot, \gamma(x))\rangle=$ $f(\gamma(x))=f_{\gamma}(x)$. So, $k_{\gamma}$ is the reproducing kernel in the time-warped space $V_{\gamma}^{2}(\varphi)$.

The above-mentioned inner product $\langle\cdot, \cdot\rangle_{\gamma}$ exists in the space $V_{\gamma}^{2}(\varphi)$. For some time-warping function $\gamma$, the following example gives an explanation of the above definition of the inner product.

EXAMPLE 3.1. Let $\gamma(w)=A w+B$, where the inner product $\langle\cdot, \cdot\rangle$ is defined as the common definition in $L^{2}\left(\mathbb{R}^{n}\right)$, where $A, B$ are $n \times n$ and $n \times 1$ constant matrices, respectively. If we define the inner product by $\left\langle f_{\gamma}, g_{\gamma}\right\rangle_{\gamma} \equiv|A|\left\langle f_{\gamma}, g_{\gamma}\right\rangle$, we have $\left\langle f_{\gamma}, g_{\gamma}\right\rangle_{\gamma}=\langle f, g\rangle$ through some simple computations.

If $\sum_{x \in X}|f(x)|^{2} \approx\|f\|_{2}^{2}$ for $X \subseteq \mathbb{R}^{d}$, then $X$ is called a set of sampling for $V^{2}(\varphi)$.

If $X$ is a set of sampling for $V^{2}(\varphi)$, then we can obtain that $\tilde{X}=\left\{\gamma^{-1}(x)\right.$ : $x \in X\}$ is a sampling space for $V_{\gamma}^{2}(\varphi)$ for the warped function $\gamma$. More exactly, we have the following proposition.

Proposition 3.2. If $X$ is a set of sampling of $V^{2}(\varphi)$ and $\gamma$ is a warped function such that $\gamma^{-1}$ is differentiable and $\left|d \gamma^{-1} / d w\right|$ is uniformly bounded, then $\tilde{X}=\left\{\gamma^{-1}(x): x \in X\right\}$ is a set of sampling for $V_{\gamma}^{2}(\varphi)$.

Proof. By the definition of the set of sampling, we know that

$$
\sum_{x \in X}\left|\left\langle f, k_{x}\right\rangle\right|^{2} \approx\|f\|_{2}^{2}
$$

Since $\gamma^{-1}$ is differentiable and $\left|d \gamma^{-1} / d w\right|$ is uniformly bounded, it is easy to check that

$$
\left\|f_{\gamma}\right\|_{2} \approx\|f\|_{2} \text {. }
$$


Combining (3.2) with (3.1), we only need to prove that

$$
\sum_{x \in X}\left|\left\langle f, k_{x}\right\rangle\right|^{2} \approx \sum_{y \in \tilde{X}}\left|\left\langle f_{\gamma}, k_{y, \gamma}\right\rangle_{\gamma}\right|^{2} .
$$

In fact,

$$
\begin{aligned}
\sum_{y \in \tilde{X}}\left|\left\langle f_{\gamma}, k_{y, \gamma}\right\rangle_{\gamma}\right|^{2} & =\sum_{y \in \tilde{X}}\left|\left\langle f_{\gamma}, k_{\gamma}(\cdot, y)\right\rangle_{\gamma}\right|^{2} \\
& =\sum_{y \in \tilde{X}}|\langle f, k(\cdot, \gamma(y))\rangle|^{2} \\
& =\sum_{x \in X}\left|\left\langle f, k\left(\cdot, \gamma\left(\gamma^{-1}(x)\right)\right)\right\rangle\right|^{2} \\
& =\sum_{x \in X}|\langle f, k(\cdot, x)\rangle|^{2} .
\end{aligned}
$$

REMARK 3.3. Similar to the proof of Proposition 3.2, if $\tilde{X}$ is a set of sampling for $V_{\gamma}^{2}(\varphi)$, then there exists $x$ such that $y=\gamma(x)$ for every $y \in \tilde{X}$, and $X=\{x\}$ is a set of sampling for the space $V^{2}(\varphi)$ for the time-warping function $\gamma$.

Proposition 3.4. If $\left\{k_{x}\right\}$ and $\left\{\tilde{k}_{x}\right\}$ are dual frames for $V^{2}(\varphi), X$ is a set of sampling for $V^{2}(\varphi)$, and $\gamma$ is a time-warped function such that $\gamma^{-1}$ is differentiable and $\left|d \gamma^{-1} / d w\right|$ is uniformly bounded, then $\left\{k_{y, \gamma}\right\}$ and $\left\{\tilde{k}_{y, \gamma}\right\}$ are dual frames for $V_{\gamma}^{2}(\varphi)$, where $k_{y, \gamma}=k_{\gamma}(\cdot, y), \tilde{k}_{y, \gamma}=\tilde{k}_{\gamma}(\cdot, y)$, and $\tilde{X}$ is the same as that in Proposition 3.2.

Proof. From the definition of dual frames, we have

$$
f=\sum_{x \in X}\left\langle f, k_{x}\right\rangle \tilde{k}_{x}=\sum_{x \in X}\left\langle f, \tilde{k}_{x}\right\rangle k_{x}
$$

By Proposition 3.2, we know that $\tilde{X}$ is a set of sampling for the time-warped space $V_{\gamma}^{2}(\varphi)$. And we have

$$
\begin{aligned}
f_{\gamma}(\cdot) & =f(\gamma(\cdot))=\sum_{x \in X}\langle f, k(\gamma(\cdot), x)\rangle \tilde{k}(\gamma(\cdot), x) \\
& =\sum_{y \in \tilde{X}}\left\langle f_{\gamma}, k_{\gamma}(\cdot, y)\right\rangle_{\gamma} \tilde{k}_{\gamma}(\cdot, y) .
\end{aligned}
$$

The second equality of the above equalities depends on the definition of the dual frames. We know the third equality from the definitions of $\tilde{X}$ and $\langle\cdot, \cdot\rangle_{\gamma}$.

Similar to (3.6), we can obtain the following:

$$
f_{\gamma}(\cdot)=\sum_{y \in \tilde{X}}\left\langle f_{\gamma}, \tilde{k}_{y}(\cdot, y)\right\rangle_{\gamma} k_{\gamma}(\cdot, y) .
$$


Combining (3.6) with (3.7), we have

$$
f_{\gamma}(\cdot)=\sum_{y \in \tilde{X}}\left\langle f_{\gamma}, \tilde{k}_{\gamma}(\cdot, y)\right\rangle_{\gamma} k_{\gamma}(\cdot, y)=\sum_{y \in \tilde{X}}\left\langle f_{\gamma}, k_{\gamma}(\cdot, y)\right\rangle_{\gamma} \tilde{k}_{\gamma}(\cdot, y) .
$$

So $k_{y, y}$ and $\tilde{k}_{y, \gamma}$ are dual frames for $V_{\gamma}^{2}(\varphi)$.

REMARK 3.5. Similar to Proposition 3.4, it is easy to see that if $\left\{k_{y, \gamma}\right\}$ and $\left\{\tilde{k}_{y, \gamma}\right\}$ are dual frames for $V_{\gamma}^{2}(\varphi)$, and $\tilde{X}$ is a set of sampling space for the timewarped space $V_{r}^{2}(\varphi)$, then there exists $x$ such that $y=\gamma(x)$ for any $y \in \tilde{X}$. So, $X \equiv\{x\}$ is a set of sampling, and $\left\{k_{x}\right\}$ and $\left\{\tilde{k}_{x}\right\}$ are dual frames for $V^{2}(\varphi)$ for the proper warping function $\gamma$.

4. Reconstruction in time-warped weighted shift-invariant spaces. In [5], Gröchenig obtained the following theorem.

THEOREM 4.1. Assume that the generator $\varphi$ satisfies the assumption (i), (ii), (iii), $m$ is $s$-moderate, and $X$ is a set of sampling for $V^{2}(\varphi)$ with dual frames $\left\{\tilde{k}_{x}\right\}(x \in X)$, then for every $f \in V_{m}^{p}(\varphi)(1 \leq p<\infty), f=\sum_{x \in X} f(x) \tilde{k}_{x}$ holds.

The following theorem gives the version of the time-warped space $V_{m, \gamma}^{p}(\varphi)$.

THEOREM 4.2. Suppose that the warped function $\gamma$ is the same as that in Proposition 3.2 or Proposition 3.4, the generator $\varphi$ satisfies assumption (i), (ii), (iii), $m$ is $s$-moderate, and $\tilde{X}$ is a set of sampling for $V_{\gamma}^{2}(\varphi)$ with dual frames $\left\{\tilde{k}_{y, \gamma}\right\}$, then for every $f_{\gamma} \in V_{m, \gamma}^{p}(\varphi)(1 \leq p<\infty), f_{\gamma}=\sum_{y \in \tilde{X}} f_{\gamma}(x) \tilde{k}_{y, \gamma}$ holds.

Proof. From Propositions 3.2 and 3.4 and Remarks 3.3 and 3.5, we know that $X=\{x: x=\gamma(y), y \in \tilde{X}\}$ is a set of sampling for $V^{2}(\varphi)$ with dual frames $\left\{\tilde{k}_{x}\right\}$.

By Theorem 4.1, for any $f \in V_{m}^{p}(\varphi)(1 \leq p<\infty)$, we have $f=\sum_{x \in X} f(x) \tilde{k}_{x}$. Accordingly, for any $f_{\gamma} \in V_{m, \gamma}^{p}(\varphi)(1 \leq p<\infty), f_{\gamma}=\sum_{y \in \tilde{X}} f_{\gamma}(x) \tilde{k}_{y, \gamma}$ holds.

It is well known that $V^{p}(\varphi)$ is $1 / 2$-band-limited signal space for $p=2$ and $\varphi=\sin (\pi x) / \pi x$. So the following example, which is the main result in [4], can be regarded as a special case or corollary of Theorem 4.2

EXAMPLE 4.3. If $\gamma$ is a time-warped function, and $T, \Omega>0$ with $0<2 T \Omega \leq 1$, then for every $g \in B_{\gamma}$,

$$
g(t)=2 T \Omega \sum g\left(\tau_{n}\right) \frac{\sin (2 \pi \Omega(\gamma(t)-n T))}{2 \pi \Omega(\gamma(t)-n T)}
$$

holds in $L^{2}(\mathbb{R})$, where $\tau_{n}:=\gamma^{-1}(n T)$ and $B_{\gamma}$ are time-warped spaces of $\Omega$ band-limited signal spaces $B \equiv\left\{f \in L^{2}(\mathbb{R}): \operatorname{supp} \hat{f} \subseteq[-\Omega, \Omega]\right\}$. 
5. Reconstruction in time-warped spline spaces. It is well known that the spline $V_{N}$ is a special case of weighted shift-invariant spaces. In [7], the following regular sampling theorem in a spline space is shown.

THEOREM 5.1. For any $f \in V_{N}$,

$$
f(x)=\sum_{n \in \mathbb{Z}} f\left(t_{n}\right) s_{n}(x),
$$

where $t_{n}=n+(N+1) / 2, s_{n}=s(\cdot-n)$, and $s$ is given by

$$
\hat{s}(w)=\frac{\hat{\varphi}_{N}(w)}{\sum_{n \in \mathbb{Z}} \varphi_{N}\left(t_{n}\right) e^{-i k w}} .
$$

As a special case of Theorem 4.1, or using the same method, we can obtain the reconstruction formula in a time-warped spline space.

THEOREM 5.2. For any $f_{\gamma} \in V_{N, \gamma}=\left\{f_{\gamma}: f_{\gamma}(\cdot)=f(\gamma(\cdot)), f \in V_{N}\right\}, f_{\gamma}(x)=$ $\sum_{\tau_{n} \in \tilde{X}} f\left(\tau_{n}\right) s_{n, \gamma}(x)$, where $\tilde{X}=\left\{\tau_{n}: \tau_{n}=\gamma^{-1}\left(t_{n}\right)\right\}, s_{n, \gamma}(\cdot)=s(\gamma(\cdot)-n), s, X$, and $\left\{t_{n}\right\}$ are the same as that in Theorem 5.1, and $\gamma$ is a time-warping function.

It is obvious that $\left\{t_{k}\right\}$ is a regular sampling, but for the proper warped function $\gamma, \tilde{X}$ and $V_{N, \gamma}$ are irregular sampling and non-band-limited signal space, respectively.

In the spline space $V_{N}$, we can construct the reproducing kernel $k_{x}$ by

$$
k(w, x)=\sum_{n} \tilde{\varphi}_{N}(w-n) \overline{\varphi_{N}(x-n)},
$$

where

$$
\tilde{\varphi}_{N}(w)=\frac{\hat{\varphi}_{N}(w)}{\left|\prod_{N}\left(e^{-i w}\right)\right|}, \quad \prod_{N}(w)=\sum_{n=0}^{2 N-2} \varphi_{2 N}(n+1) w^{k} .
$$

In fact, from [8], we know that $\left\langle\varphi_{N}(\cdot-m), \tilde{\varphi}_{N}(\cdot-n)\right\rangle=\delta_{m n}$. Using this fact, it is easy to check that $\langle f, k(\cdot, x)\rangle=f(x)$.

We will give another proof for Theorem 5.2 below. First, we show the following theorem that already has been given in [9].

THEOREM 5.3. Suppose $S$ is a reproducing kernel Hilbert space (RKHS) with inner product $\langle\cdot, \cdot\rangle$, then a sampling basis $\left\{\varphi_{n}\right\}$ of $S$ yields a reconstruction formula of the form

$$
f(t)=\sum_{n} f\left(t_{n}\right) \varphi_{n}(t)
$$

from a sampling set $\left\{t_{n}\right\}$ if and only if its biorthogonal basis $\left\{\tilde{\varphi}_{n}\right\}$ is given by $\tilde{\varphi}_{n}(x)=k\left(t_{n}, x\right)$.

Using Theorem 5.3, we can prove Theorem 5.2. 
In fact, by the discussion of Section 2, we know that $V_{N, \gamma}$ is an RKHS with the reproducing kernel $k_{x, y}$ given in the above part of this section and the inner product $\langle\cdot, \cdot\rangle_{\gamma}$ defined in Section 2 . So, we only need to prove the biorthogonal relation between $s_{n, y}$ and $k_{\gamma}\left(\tau_{n}, x\right)$.

It is easy to know that

$$
\left\langle k_{\gamma}\left(\tau_{n}, \cdot\right), s_{m, \gamma}(\cdot)\right\rangle_{\gamma}=\left\langle k\left(\gamma\left(\tau_{n}\right), \cdot\right), s_{m}(\cdot)\right\rangle=\left\langle k\left(t_{n}, \cdot\right), s_{m}(\cdot)\right\rangle .
$$

At the same time, from Theorem 5.1 and the necessary conditions of Theorem 5.3 , we can imply the biorthogonal relation between $\left\{s_{n}(\cdot)\right\}$ and $\left\{k\left(t_{n}, \cdot\right)\right\}$. So

$$
\left\langle k_{\gamma}\left(\tau_{n}, \cdot\right), s_{m, \gamma}(\cdot)\right\rangle_{\gamma}=\left\langle k\left(t_{n}, \cdot\right), s_{m}(\cdot)\right\rangle=\delta_{m n},
$$

that is, $\left\{k_{\gamma}\left(\tau_{n}, \cdot\right)\right\}$ is a biorthogonal basis of $\left\{s_{n, \gamma}\right\}$. Then by the sufficient condition of Theorem 5.3, we can obtain Theorem 5.2.

ACKNOWLEDGMENT. The authors gratefully thank the referees and the editor for valuable suggestions which helped improving this paper.

\section{REFERENCES}

[1] A. Aldroubi and K. Gröchenig, Beurling-Landau-type theorems for non-uniform sampling in shift invariant spline spaces, J. Fourier Anal. Appl. 6 (2000), no. 1, 93-103.

[2] _ Nonuniform sampling and reconstruction in shift-invariant spaces, SIAM Rev. 43 (2001), no. 4, 585-620.

[3] S. Azizi, D. Cochran, and J. N. McDonald, Reproducing kernel structure and sampling on time-warped spaces with application to warped wavelets, IEEE Trans. Inform. Theory 48 (2002), no. 3, 789-790.

[4] J. J. Clark, M. R. Palmer, and P. D. Lawrence, A transformation method for the reconstruction of functions from nonuniformly spaced samples, IEEE Trans. Acoust. Speech Signal Process. 33 (1985), no. 4, 1151-1165.

[5] K. Gröchenig, Localization of frames, Banach frames, and the invertibility of the frame operator, to appear in J. Fourier Anal. Appl.

[6] Y. Liu, Irregular sampling for spline wavelet subspaces, IEEE Trans. Inform. Theory 42 (1996), no. 2, 623-627.

[7] W. Sun and X. Zhou, Average sampling in spline subspaces, Appl. Math. Lett. 15 (2002), no. 2, 233-237.

[8] J. Wang, Spline wavelets in numerical resolution of partial differential equations, Wavelet Analysis and Applications (Guangzhou, 1999), AMS/IP Stud. Adv. Math., vol. 25, American Mathematical Society, Rhode Island, 2002, pp. 257277.

[9] A. I. Zayed, Advances in Shannon's Sampling Theory, CRC Press, Florida, 1993.

Jun Xian: Department of Mathematics, Sun Yat-Sen University, Guangzhou 510275, China

E-mail address: xi anjun11@sohu.com

Yongjin Li: Department of Mathematics, Sun Yat-Sen University, Guangzhou 510275, China

E-mail address: sts7yj@zsu.edu.cn 


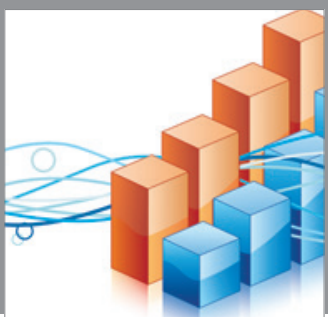

Advances in

Operations Research

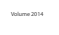

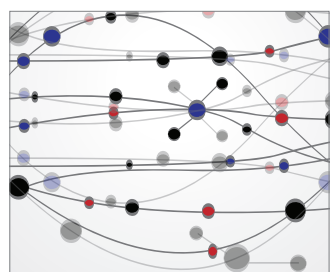

\section{The Scientific} World Journal
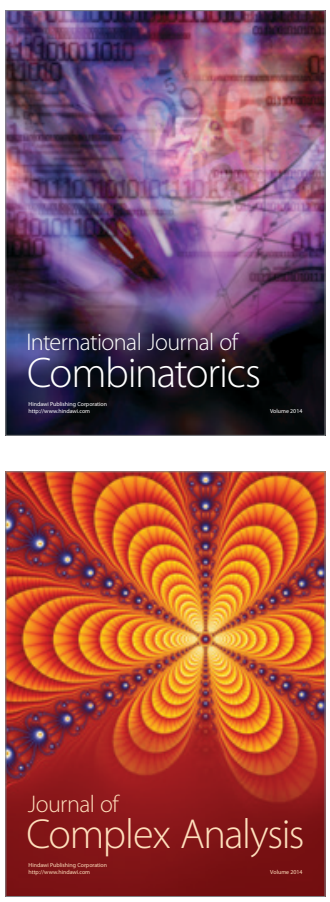

International Journal of

Mathematics and

Mathematical

Sciences
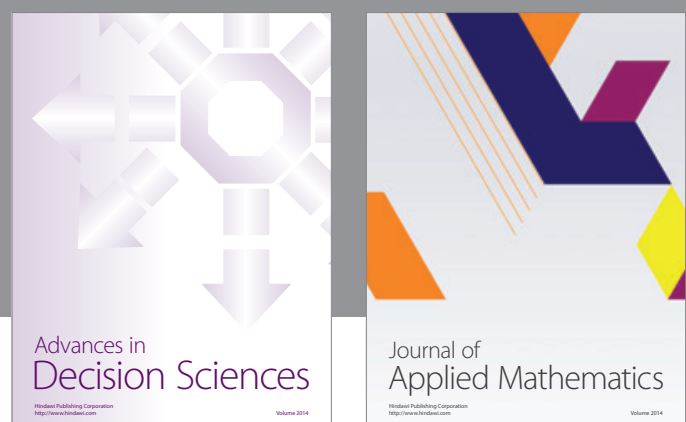

Journal of

Applied Mathematics
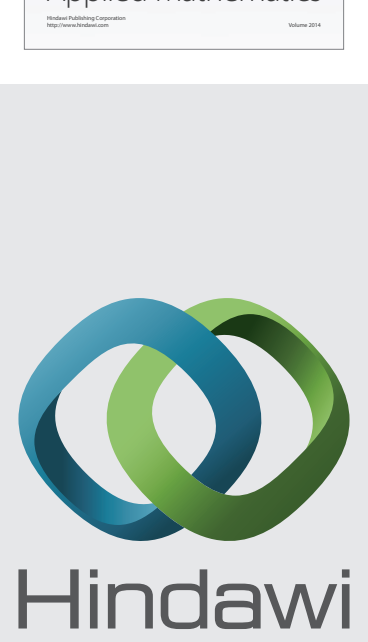

Submit your manuscripts at http://www.hindawi.com
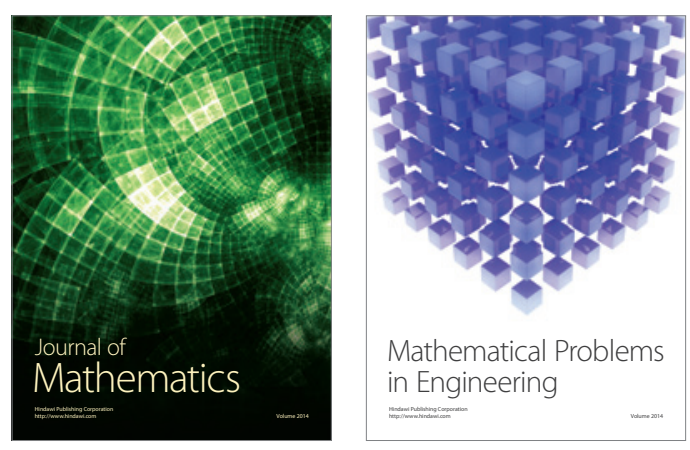

Mathematical Problems in Engineering
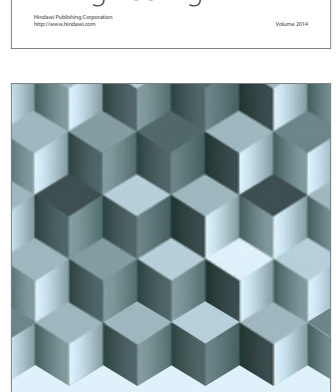

Journal of

Function Spaces
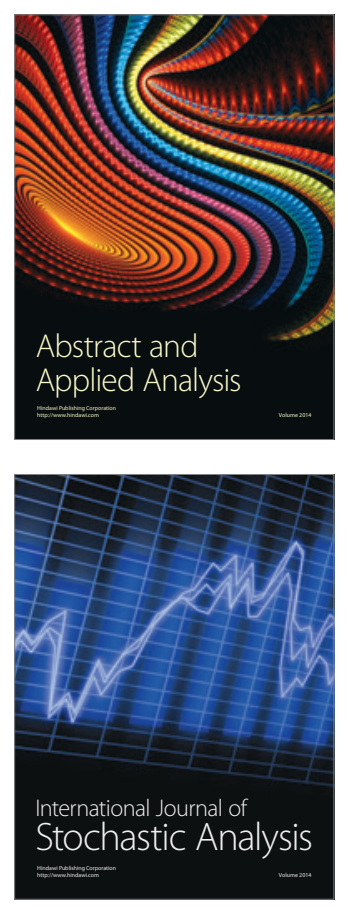

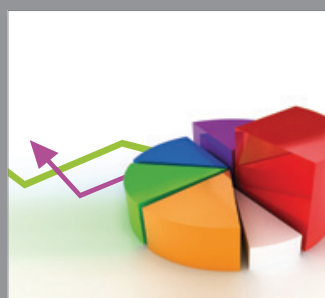

ournal of

Probability and Statistics

Promensencen
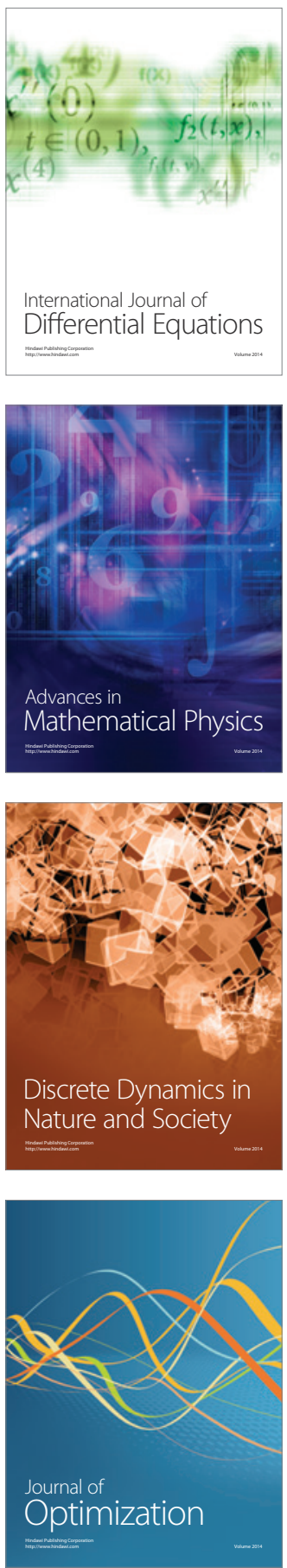